\title{
Risk perception is not associated with attendance at a preventive intervention for type 2 diabetes mellitus among South Asians at risk of diabetes
}

\author{
Everlina MA Vlaar ${ }^{1, *}$, Vera Nierkens ${ }^{1}$, Mary Nicolaou ${ }^{1}$, Barend JC Middelkoop ${ }^{2,3}$, \\ Karien Stronks ${ }^{1}$ and Irene GM van Valkengoed ${ }^{1}$ \\ 'Department of Public Health, Academic Medical Centre, University of Amsterdam, Meibergdreef 9, K2.204-1, \\ 1105 AZ Amsterdam, The Netherlands: ${ }^{2}$ Department of Public Health, Leiden University Medical Centre, Leiden, \\ The Netherlands: ${ }^{3}$ Public Health Service, The Hague, The Netherlands
}

Submitted 8 August 2013: Final revision received 28 April 2014: Accepted 2 May 2014: First published online 10 June 2014

\begin{abstract}
Objective: To evaluate the association between risk perception and attendance in a diabetes prevention programme among South Asians with a high risk for diabetes. Design: An observational study. We measured risk perception during the baseline interview with causal beliefs, perceived susceptibility and perceived controllability. We used logistic regression to examine the relationship between risk perception and attendance. We adjusted for relevant sociodemographic factors, screening results and psychosocial factors.

Setting: The Hague, the Netherlands.

Subjects: Five hundred and thirty-five Hindustani Surinamese (South Asians) aged 18-60 years from a lifestyle-versus-control intervention for the prevention of diabetes.

Results: In total, $68.2 \%$ attended the lifestyle or control intervention. Participants perceived lifestyle and heredity to increase the risk of diabetes and perceived increasing physical activity to decrease it. Only $44.2 \%$ of the participants perceived themselves as susceptible to diabetes and only those who perceived a family history of diabetes as a cause of diabetes appeared to be more inclined to attend. However, after adjustment for confounding, the association was not statistically significant.

Conclusions: Risk perception was not significantly associated with attendance. The results suggest that increasing the risk perception alone in this South Asian population is unlikely to increase the attendance at a diabetes prevention programme.
\end{abstract}

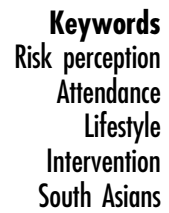

South Asian migrants and their offspring living in industrialized countries (henceforth 'South Asians') are at high risk of developing type 2 diabetes mellitus ${ }^{(1-4)}$. In the Netherlands, the prevalence among the Hindustani Surinamese, who are of South Asian origin, is about four times greater than the prevalence among the ethnic Dutch $^{(4)}$. Given this high risk of diabetes, efforts have been made to develop lifestyle interventions that are culturally targeted to the South Asian population at risk ${ }^{(5,6)}$. In practice, the effectiveness of such interventions will depend on whether those diagnosed as being at high risk attend the counselling that is offered.

Previous studies among patient groups have found that increased risk perception enhances attendance at an intervention $^{(7,8)}$. Risk perception (Fig. 1) can be defined as the subjective assessment of the probability that someone will get a certain disease (susceptibility) and how concerned someone is with the consequences (severity) ${ }^{(9-11)}$. Strategies to increase risk perception, particularly communication about common risk factors, are commonly used to enhance attendance at lifestyle interventions. However, whether a similar association of risk perception with attendance can be assumed for South Asian populations, as was done for patient groups in previous studies, has not been investigated. At the same time, there are reasons to expect this association to be different from associations in other, generally lower-risk populations.

First, because a family history of diabetes is more common among South Asian populations than other populations ${ }^{(4)}$, South Asians are expected to perceive 


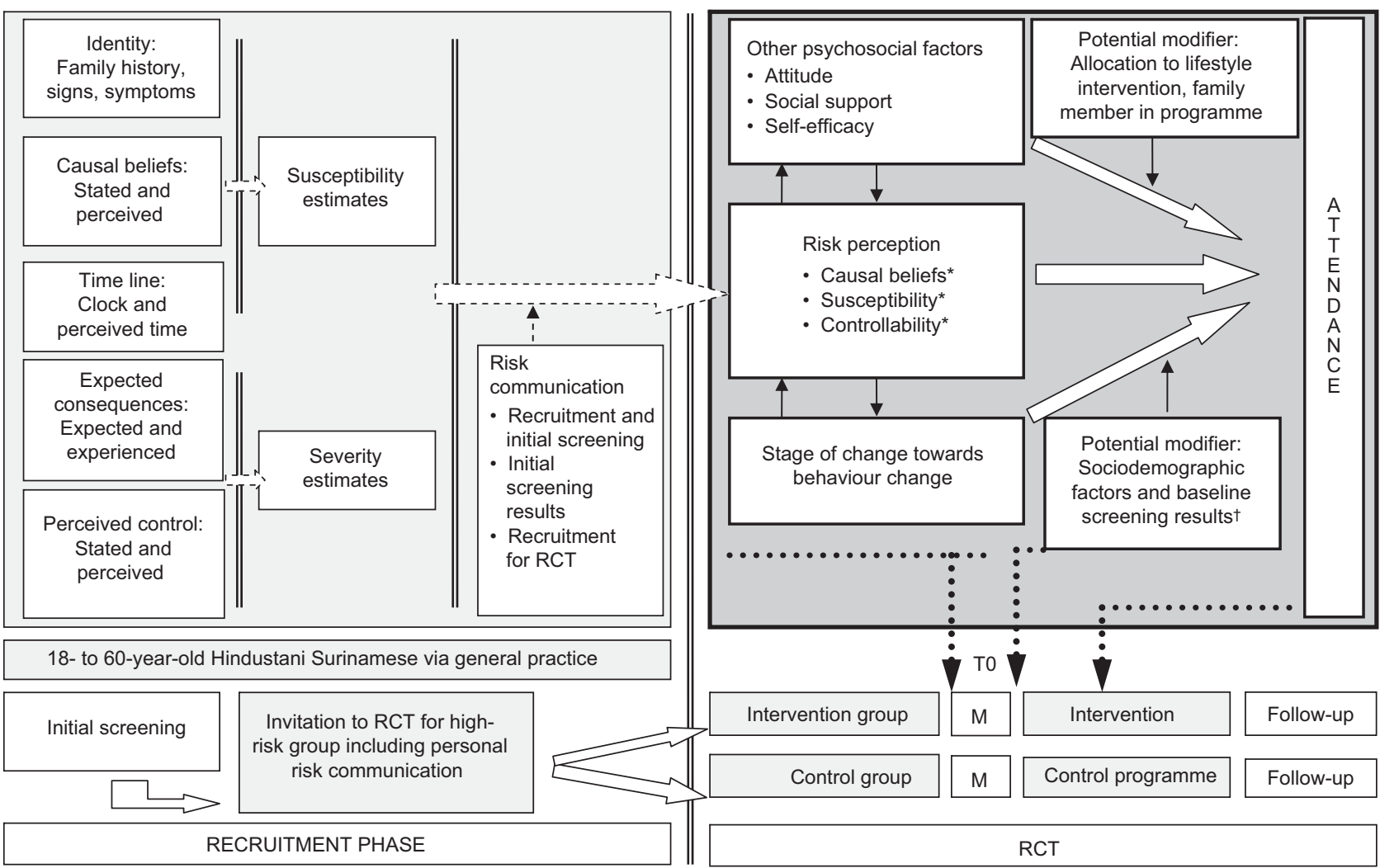

Fig. 1 Conceptual model and implementation of the measurements in the study design (M, measurements; RCT, randomized controlled trial). *Measured items of risk perception: causal beliefs consisted of three items, i.e. heredity and general and groupspecific lifestyle factors; susceptibility was an overall item and controllability was only measured for physical activity ${ }^{(9)}$. †The results of the screening were communicated to the participants.

themselves as more frequently susceptible than the general population $^{(11-13)}$. A family history of diabetes is thought to increase the awareness of one's own risk and, therefore, to motivate one to attend a lifestyle intervention. If, however, most of the population is aware of the increased risk, this might decrease the importance of focusing on risk perception to improve attendance.

Second, due to this frequent family history of diabetes in combination with the high prevalence of diabetes in the community, heredity may be considered more often than in other populations to be a more likely cause of diabetes than an unhealthy lifestyle ${ }^{(5,13)}$. On the one hand, such heredity beliefs may trigger participation in a lifestyle programme $e^{(14-16)}$. On the other hand, these heredity beliefs may cause a feeling of inevitability and a perception of little controllability ${ }^{(5,16-20)}$. If this is the case, it might lead to non-attendance at a lifestyle programme. Fatalism, which is related to heredity beliefs and has been observed in the South Asian population, might reinforce this behaviour ${ }^{(5,16,20,21)}$.

Finally, causal beliefs about the risk of diabetes in the South Asian population may differ from the generally measured causes ${ }^{(5,22)}$. For example, participants in focus group discussions mentioned the traditional meals as a cause of diabetes and, in particular, the traditional use of large amounts of oil, white rice or masala (spices) ${ }^{(5)}$. Such group-specific causal beliefs were also seen as an important cause of the onset of diabetes in a South Asian population in Norway ${ }^{(22)}$. These beliefs may be more strongly related to attendance than general causal beliefs.

In the current study we aimed to assess risk perception and its association with attendance at a diabetes lifestyle intervention (focusing on diet and physical activity (PA)) or a control intervention for 18- to 60-year-old Hindustani Surinamese (of South Asian ancestry) at high risk of diabetes in a randomized controlled trial in The Hague, the Netherlands. Specifically, we assessed: (i) the extent to which a risk of diabetes is perceived and the causal beliefs, i.e. general lifestyle beliefs (perceiving general risk factors as causes of diabetes), group-specific lifestyle beliefs, heredity beliefs, perceived susceptibility and perceived controllability by PA; and (ii) the association of these constructs with attendance.

\section{Experimental methods}

\section{Study population}

The study population consisted of participants in the DH!AAN study, a randomized controlled trial (Trial number 
Box 1 Additional information about the origin of the Hindustani Surinamese

The term 'Hindustani Surinamese' refers to people of South Asian ancestral origin and their offspring who migrated to the Netherlands via Suriname. The Hindustani Surinamese are the descendants of the labourers from North India - Uttar Pradesh, Uttaranchal and West Bihar - who were indentured between 1873 and $1917^{(23)}$.

NTR1499) designed to study the effectiveness of a culturally targeted, intensive lifestyle intervention for the prevention of diabetes and cardiovascular risk factors among the Hindustani Surinamese (South Asians; Box 1) at high risk of diabetes ${ }^{(24)}$. The study was conducted according to the Medical Ethics Committee of the Academic Medical Center of Amsterdam. Written informed consent was obtained from all participants.

\section{Study design}

Details of the DH!AAN study have been published elsewhere $^{(24)}$. Briefly, South Asian adults aged 18-60 years were selected from forty-eight general practice lists in The Hague. People with known diabetes and pregnant women were excluded. All potential participants received an invitation for an initial screening for diabetes risk (Fig. 1). The participants in the initial screening were informed of their screening results. Subsequently, those at high risk of diabetes received an invitation to take part in the DH!AAN trial. This included persons with impaired fasting glucose (fasting plasma glucose of 5.6-6.9 mmol/1), impaired glucose tolerance (2-h post-load glucose of $7 \cdot 8-11 \cdot 0 \mathrm{mmol} / \mathrm{l}$ ), a glycated $\mathrm{Hb}$ (HbA1c) level of $42 \mathrm{mmol} / \mathrm{mol}$ or more, and/or a homeostasis model assessment of estimated insulin resistance (HOMA-IR) of 2.39 or more.

The letter of invitation to the trial contained general information about the trial, i.e. information about the random assignment to one of the lifestyle interventions and the planned measurements at baseline and after 1 and 2 years. The invitation was followed by a telephone call from a member of the study team. We randomly allocated invitees who provided informed consent to either the lifestyle intervention or the control intervention. Details about the allocated intervention were given after the baseline measurement. In total, we recruited 536 people who completed the baseline measurement between 18 May 2009 and 11 October 2010 (24).

\section{Lifestyle intervention}

After the baseline measurement, we invited the participants allocated to the lifestyle group to the intervention, which consisted of culturally targeted individual lifestyle counselling. This could be supplemented with a family session, two group-based cooking classes and a supervised PA programme, as described previously ${ }^{(5,24)}$. All participants were contacted by letter and by telephone and were referred to a study dietitian near their place of residence. The dietitian was given the participant's contact information and was instructed to contact the participant directly to make an appointment. If the dietitian had not reached the participant after three telephone attempts, the study team sent a letter to ask the participant to contact the dietitian's office.

\section{Control intervention}

After the baseline measurement, the control group was offered two group sessions (after the baseline measurements and after 6 months) in which they received generic information about diabetes, the current guidelines for diet and PA, and methods for achieving the recommended levels of $\mathrm{PA}^{(24)}$. We invited all participants to the programme by letter and by telephone. During the telephone call, the participants were given the opportunity to make an appointment for the first group session. Those who we did not reach with three attempts received a letter requesting them to call the study team for an appointment.

\section{Data collection}

The baseline and follow-up measures of the trial each included a structured face-to-face interview, a physical examination and blood tests. We assessed the levels of fasting glucose (hexokinase method; Roche Diagnostics), 2-h post-load glucose (oral glucose tolerance test (OGTT) with glucose load $75 \mathrm{~g}$, glucose determined by the hexokinase method; Roche Diagnostics), insulin (immunoassay, sandwich principle; Roche Diagnostics) and HbA1c (HPLC). The results of their blood tests and the physical examination were given to the participants (Fig. 1).

\section{Measurements and definitions}

\section{Attendance}

We defined attendance at the lifestyle intervention as having attended at least one counselling session as reported in the dietitians' registration files. In the control intervention, attendance was defined as having attended the first group session as reported in the student dietitian's registration. Because the student dietitian had incompletely registered attendance for some sessions, we supplemented the data for the control group with self-reported attendance and verified these combined data with our own study administration of the appointments. If a mismatch for a participant in the control group was found, attendance data were considered missing. On this basis, one participant was excluded, leaving the data for 535 of the 536 participants for analyses.

\section{Risk perception}

Risk perception was measured during the baseline interview (Fig. 1). We measured perceived susceptibility and 
two components of Leventhal's Common-Sense Model of illness representation and self-regulation ${ }^{(25)}$; i.e. causal beliefs and perceived controllability by PA (Fig. 1).

Causal beliefs were measured with twelve statements about the perceived influence on the onset of diabetes of certain behaviours or characteristics on a 3-point Likert scale. The statements concerned: (i) general lifestyle beliefs related to seven general risk factors of diabetes (e.g. 'Are you at high risk of diabetes when you are overweight?'); and (ii) three group-specific lifestyle beliefs (e.g. 'Are you at high risk of diabetes when you eat masala?') derived from our focus group discussions ${ }^{(5)}$. It is relevant to note that not the correctness of the statement, but rather that the existence of the belief was important. The third question (iii) concerned two statements about heredity (e.g. 'Are you at high risk of diabetes if you have a family member with diabetes?') ${ }^{(26,27)}$. The beliefs related to overweight, unhealthy food, little exercise, hypertension, age and smoking were combined into a single factor based on the results of internal consistency analysis ( $n$ 6, $\alpha=0.63$ ). When the score was at least 4 of 6 , participants were classed as 'perceiving general lifestyle as a cause of diabetes'. We excluded one statement, concerning the perception of gestational diabetes, from the analysis due to the many missing values.

Perceived susceptibility was measured with a three-item perceived susceptibility score on a 5-point Likert scale ( $n$ 3, $\alpha=0.63)^{(14)}$. The items were dichotomized into high susceptibility (positive score) and low susceptibility (negative or neutral value as a reference).

Perceived controllability was measured with a one-item score on a 5-point Likert scale ('Do you believe that PA lowers your chances of developing diabetes?'), and it was dichotomized similarly to perceived susceptibility.

\section{Potential confounders and effect modifiers}

We identified sociodemographic factors (age, gender, education and family history of diabetes in first- or seconddegree relative), screening results at baseline (a high OGTT result at baseline and a high BMI), other psychosocial factors and the stage of change towards behaviour change as potential confounders in our analyses. This was based on previous studies, behaviour change models and the mental model of being at risk (Fig. 1) ${ }^{(7,8,28-30)}$. Moreover, we also considered sociodemographic factors, screening results at baseline, allocation to the control intervention and having a family member in the trial as potential effect modifiers ${ }^{(31,32)}$. The online supplementary material details the measurement of these factors, and we present them briefly here.

Screening results at baseline. We selected the parameters from the results of baseline measurements that we communicated to the participants. We calculated BMI as weight $(\mathrm{kg}) /$ height $(\mathrm{m})^{2}$. We classified blood test results on the basis of the fasting and 2-h post-load glucose values from the OGTT, the HbA1c value and the HOMA-IR.
We calculated the HOMA-IR as glucose (mmol/l) multiplied by fasting insulin $(\mathrm{mU} / \mathrm{l})$ divided by $22 \cdot 5^{(33)}$. One group represented participants with 'a high OGTT result at baseline' (impaired fasting glucose and/or impaired glucose tolerance) and one group represented participants with 'no high OGTT result at baseline' (e.g. those with only an elevated HbA1c level and/or elevated HOMA-IR). As the OGTT was the only recommended diagnostic option until recently ${ }^{(34)}$, we expected that it might provide a more recognizable risk marker for the participants than the other measures and that this could influence the attendance.

Other psychosocial factors. We addressed these factors in the baseline questionnaire. Internal consistency analysis resulted in three factors for attitudes towards PA and diet: direct ( $n$ 4, $\alpha=0.64)$ and indirect attitudes towards PA ( $n 7, \alpha=0.67$ ) and attitude towards conventional healthy diet behaviours ( $n$ 10, $\alpha=0 \cdot 84$ ). Two factors were formed for social support: perceived social support for PA from other family members and close relatives ( $n 2, \alpha=0.68$ ) and perceived social support for conventional healthy diet behaviours ( $n$ 5, $\alpha=0.94$ ).

Self-efficacy was reflected in two combined factors: perceived self-efficacy for PA ( $n 5, \alpha=0.73)$ and perceived self-efficacy for conventional healthy diet behaviours ( $n$ 5, $\alpha=0.66$ ). Due to empty answer categories, we dichotomized the resulting variables (a positive value $v$. a negative or neutral value as reference).

The stages of change towards diet and PA were classed as being motivated or unmotivated to change the diet according to the Dutch guidelines and PA within 6 months $^{(24)}$. The stage of change towards diet was measured for each specific diet behaviour. One factor was formed for stage of change towards conventional healthy diet behaviours ( $n 5, \alpha=0 \cdot 73$ ).

\section{Statistical analyses}

First, we used means with standard deviations or numbers and percentages to describe the demographic characteristics, screening results, stage of change and other psychosocial factors in the total population, which we stratified by attendance. We then used independent-sample $t$ tests and Mann-Whitney $U$ tests to analyse differences between the attendees and non-attendees in sociodemographic characteristics, allocation to the lifestyle or control intervention, screening results, stage of change and all other psychosocial factors. To limit the number of potential confounders in the analyses, we selected only those with $P<0.25$ for the association with attendance as potential confounders for the further analyses.

Second, we described the prevalence of the risk perception parameters and determined the univariate association between these parameters and attendance at the lifestyle or control intervention by means of logistic regression analysis.

Third, we determined whether the selected potential confounders attenuated the association between risk 
Table 1 Baseline characteristics of the study sample; Hindustani Surinamese (South Asians) aged 18-60 years participating in a diabetes lifestyle or control intervention for South Asians in The Hague, the Netherlands (DH!AAN study)

\begin{tabular}{|c|c|c|c|c|c|c|c|}
\hline & \multicolumn{7}{|c|}{ Attendance } \\
\hline & \multicolumn{2}{|c|}{$\begin{array}{l}\text { Total } \\
(n 535)\end{array}$} & \multicolumn{2}{|c|}{$\begin{array}{l}\text { Non-attendees } \\
\quad(n 174)\end{array}$} & \multicolumn{2}{|c|}{$\begin{array}{l}\text { Attendees } \\
\text { (n 361) }\end{array}$} & \multirow[b]{2}{*}{$P$} \\
\hline & $n$ or Mean & $\%$ or SD & $n$ or Mean & $\%$ or SD & $n$ or Mean & $\%$ or SD & \\
\hline \multicolumn{8}{|l|}{ Sociodemographic characteristics } \\
\hline Number of men & 265 & 49.5 & 92 & $54 \cdot 1$ & 173 & 47.4 & 0.148 \\
\hline Mean age in years (SD) & 43.5 & $10 \cdot 4$ & $42 \cdot 2$ & $10 \cdot 6$ & $44 \cdot 2$ & $10 \cdot 2$ & 0.037 \\
\hline High level of education* & 458 & 88.2 & 145 & 87.3 & 313 & 88.7 & 0.664 \\
\hline Family history of diabetes & 397 & $76 \cdot 2$ & 130 & $78 \cdot 3$ & 267 & $75 \cdot 2$ & 0.439 \\
\hline Family member in trial & 49 & $9 \cdot 2$ & 14 & $8 \cdot 2$ & 35 & $9 \cdot 6$ & 0.614 \\
\hline Allocated to control intervention & 252 & $47 \cdot 1$ & 82 & $48 \cdot 2$ & 170 & $46 \cdot 6$ & 0.720 \\
\hline \multicolumn{8}{|l|}{ Screening results } \\
\hline Mean $\mathrm{BMI}$ in $\mathrm{kg} / \mathrm{m}^{2}(\mathrm{sD})$ & $27 \cdot 5$ & 4.1 & $27 \cdot 2$ & 4.1 & $27 \cdot 6$ & 4.2 & 0.343 \\
\hline Blood test: high OGTT values† & 205 & 38.5 & 51 & 29.7 & 154 & $42 \cdot 7$ & 0.009 \\
\hline \multicolumn{8}{|l|}{ Other psychosocial factors } \\
\hline \multicolumn{8}{|l|}{ Positive attitude } \\
\hline \multicolumn{8}{|l|}{ PA } \\
\hline Direct & 509 & $97 \cdot 3$ & 163 & 98.8 & 346 & $96 \cdot 6$ & 0.159 \\
\hline Indirect & 505 & $96 \cdot 6$ & 163 & 98.8 & 342 & 95.5 & 0.058 \\
\hline \multicolumn{8}{|l|}{ Diet } \\
\hline Conventional healthy diet & 513 & 98.3 & 163 & 98.8 & 350 & $98 \cdot 0$ & 0.542 \\
\hline Importance of brown rice & 219 & 41.9 & 60 & $36 \cdot 4$ & 159 & 44.4 & 0.083 \\
\hline Importance of snacks & 150 & $29 \cdot 2$ & 44 & $27 \cdot 3$ & 106 & $30 \cdot 0$ & 0.533 \\
\hline Pleasure in brown rice & 127 & $25 \cdot 0$ & 42 & $26 \cdot 4$ & 85 & 24.4 & 0.632 \\
\hline Pleasure in snacks & 270 & 52.9 & 84 & $52 \cdot 8$ & 186 & 53.0 & 0.973 \\
\hline \multirow{2}{*}{\multicolumn{8}{|c|}{$\begin{array}{l}\text { Perceived social support } \\
\text { PA }\end{array}$}} \\
\hline & & & & & & & \\
\hline Partner & 246 & $47 \cdot 0$ & 81 & $49 \cdot 1$ & 165 & $46 \cdot 1$ & 0.523 \\
\hline Others & 339 & $64 \cdot 8$ & 105 & 63.6 & 234 & $65 \cdot 4$ & 0.701 \\
\hline \multicolumn{8}{|l|}{ Diet } \\
\hline Conventional healthy diet & 300 & $57 \cdot 6$ & 97 & $58 \cdot 8$ & 203 & $57 \cdot 0$ & 0.705 \\
\hline Brown rice & 132 & $25 \cdot 3$ & 31 & $18 \cdot 8$ & 101 & 28.3 & 0.020 \\
\hline Snacks & 147 & 28.2 & 43 & $26 \cdot 1$ & 104 & $29 \cdot 1$ & 0.469 \\
\hline \multicolumn{8}{|l|}{ Perceived self-efficacy } \\
\hline PA & 358 & 68.5 & 120 & $72 \cdot 7$ & 238 & 66.5 & 0.154 \\
\hline \multicolumn{8}{|l|}{ Diet } \\
\hline Conventional healthy diet & 483 & 92.4 & 157 & $95 \cdot 2$ & 326 & $91 \cdot 1$ & 0.102 \\
\hline Brown rice & 159 & 30.5 & 59 & 35.8 & 100 & $28 \cdot 0$ & 0.074 \\
\hline Snacks & 319 & 62.5 & 101 & 63.5 & 218 & $62 \cdot 1$ & 0.760 \\
\hline \multicolumn{8}{|l|}{ Stage of change } \\
\hline \multicolumn{8}{|l|}{ Motivated to change/adopt } \\
\hline PA & 351 & $65 \cdot 6$ & 98 & $57 \cdot 6$ & 253 & $69 \cdot 3$ & 0.008 \\
\hline \multicolumn{8}{|l|}{ Diet } \\
\hline Conventional healthy diet & 475 & 91.0 & 150 & $90 \cdot 9$ & 325 & 91.0 & 0.962 \\
\hline Brown rice & 226 & 43.3 & 71 & 43.0 & 155 & 43.4 & 0.934 \\
\hline
\end{tabular}

OGTT, oral glucose tolerance test; PA, physical activity.

Data are presented as $n$ and \% unless otherwise indicated.

Differences in baseline characteristics were calculated with independent-sample $t$ tests (continuous data) and Mann-Whitney $U$ tests (binary data).

*Education was classified as low (low vocational training or lower secondary education) or high (intermediate vocational training and higher secondary education to higher vocational training or university).

†The blood test results were classed for participants with 'a high OGTT result at baseline' (impaired fasting glucose and/or impaired glucose tolerance) and participants with 'no high OGTT result at baseline' (e.g. only an elevated glycated Hb level and/or an elevated homeostasis model assessment of estimated insulin resistance).

perception and participation. Then we determined whether sociodemographic factors, screening results at baseline, allocation to the control intervention or having a family member in the trial modified the association of the risk perception factors with attendance.

Finally, we carried out a sensitivity analysis to determine whether the potential dependency between participants belonging to the same general practice influenced the association between risk perception and attendance in the final model. We did not find evidence of differences between practices (data not shown). Given the small number of participants with members from the same household in the study ( $n$ 49), we refrained from carrying out a multilevel analysis with a level to account for household.

For all analyses, with the exception of the selection of the potential confounders, we considered $P<0.05$ as statistically significant. We used the statistical software package IBM SPSS Statistics $19 \cdot 0$ for the analyses. 


\section{Results}

\section{Characteristics of the population and attendance}

Of all participants included in our analyses ( $n$ 535), $49.5 \%$ were men, the mean age was 43.5 (sD 10.4) years, and $11 \cdot 8 \%$ had a low level of education. Moreover, $76 \cdot 2 \%$ had a family history of diabetes, and the mean BMI was $27 \cdot 5$ (sD $4 \cdot 1$ ) $\mathrm{kg} / \mathrm{m}^{2}$ (Table 1). Approximately two-thirds of the participants ( $n$ 361, 68.2\%) attended the lifestyle intervention or the control intervention. Attendees were older and more frequently had a higher OGTT result at baseline than non-attendees.

\section{Risk perception}

Most participants perceived the general lifestyle factors, consuming large amounts of sugar and white rice (but not masala) as causes of the onset of diabetes (Table 2). Moreover, although $72.5 \%$ perceived 'being a South Asian' (heredity) as a cause of diabetes and $88.9 \%$ perceived a 'family history of diabetes' (heredity) as a cause, 87.0\% believed that one can control diabetes by being physically active. In addition, less than half of our study population ( $44.2 \%$ ) perceived themselves as susceptible to the onset of diabetes.

\section{Relationship of risk perception parameters to attendance}

In general, attendees more frequently reported the causal beliefs, perceived high susceptibility and perceived controllability by PA than non-attendees (Table 2). For instance, attendees reported perceiving a family history of diabetes as a cause of diabetes more frequently than non-attendees did, and more often perceived themselves as susceptible to the onset of diabetes. However, all these factors, except perceiving a family history of diabetes as a cause of diabetes (OR $=1 \cdot 76 ; 95 \%$ CI 1.01, 3.07), were not significantly associated with attendance.

On the basis of the criterion of $P<0.25$ for the association with attendance, we selected the perceived attitude towards PA, replacing white rice with brown rice, selfefficacy, social support regarding the consumption of brown rice, and motivation to increase PA as potential confounders in the further analysis (Table 1). The OR estimates for the association of the risk perception parameters with attendance, although not statistically significant, remained comparable after adjustment for all risk perception parameters and potential confounders (Table 3). We found no evidence for effect modification by age, gender, education, family history of diabetes, allocation to the control intervention, having a family member in the trial, a high OGTT result at baseline or a high BMI.

\section{Discussion}

The attendance of our South Asian population at the lifestyle or control intervention was comparable with the attendance at lifestyle interventions in studies among other ethnic and high-risk populations ${ }^{(35-38)}$. Moreover, our South Asian population had several perceptions that were similar to those of other populations at increased risk of diabetes ${ }^{(8,13,17,39-43)}$. In contrast, a South Asian population in Norway less frequently reported perceiving the influence of general lifestyle and heredity than our population $^{(22)}$. This might be related to the Norwegian South Asian population's level of education being lower

Table 2 The univariate association of risk perception with attendance at a diabetes lifestyle or control intervention for South Asians among Hindustani Surinamese (South Asians) aged 18-60 years in The Hague, the Netherlands (DH!AAN study)

\begin{tabular}{|c|c|c|c|c|c|c|c|c|c|}
\hline \multirow[b]{3}{*}{ Risk perception } & \multicolumn{6}{|c|}{ Attendance } & \multirow[b]{3}{*}{ OR } & \multirow[b]{3}{*}{$95 \% \mathrm{Cl}$} & \multirow[b]{3}{*}{$P$} \\
\hline & \multicolumn{2}{|c|}{$\begin{array}{l}\text { Total } \\
\text { (n 535) }\end{array}$} & \multicolumn{2}{|c|}{$\begin{array}{l}\text { Non-attendees } \\
\quad(n 174)\end{array}$} & \multicolumn{2}{|c|}{$\begin{array}{l}\text { Attendees } \\
\text { (n 361) }\end{array}$} & & & \\
\hline & $n$ & $\%$ & $n$ & $\%$ & $n$ & $\%$ & & & \\
\hline \multicolumn{10}{|l|}{ Causal beliefs: general lifestyle factors } \\
\hline Perceived general lifestyle factors as causes ${ }^{\star}$ & 364 & $69 \cdot 6$ & 110 & $66 \cdot 7$ & 254 & $70 \cdot 9$ & 1.22 & $0.82,1.81$ & 0.323 \\
\hline \multicolumn{10}{|l|}{ Causal beliefs: group-specific lifestyle factors } \\
\hline Perceived consumption of much sugar as a cause & 374 & 71.5 & 115 & $69 \cdot 7$ & 259 & $72 \cdot 3$ & 1.14 & $0.76,1.31$ & 0.533 \\
\hline Perceived white rice as a cause & 307 & 58.7 & 95 & $57 \cdot 6$ & 212 & $59 \cdot 2$ & 1.07 & $0.74,1.56$ & 0.723 \\
\hline Perceived masala as a cause & 173 & 33.2 & 51 & 30.9 & 122 & $34 . \overline{3}$ & 1.17 & $0.78,1.73$ & 0.449 \\
\hline \multicolumn{10}{|l|}{ Causal beliefs: heredity } \\
\hline Perceived being a South Asian as a cause & 379 & 72.5 & 115 & 69.7 & 264 & 73.7 & 1.22 & $0.81,1.83$ & 0.336 \\
\hline Perceived family history of DM as a cause & 465 & 88.9 & 140 & 84.8 & 325 & $90 \cdot 8$ & 1.76 & $1.01,3.07$ & 0.047 \\
\hline \multicolumn{10}{|l|}{ Perceived susceptibility } \\
\hline Perceived themselves to be susceptible & 231 & $44 \cdot 2$ & 63 & 38.2 & 168 & $46 \cdot 9$ & 1.43 & $0.98,2.09$ & 0.062 \\
\hline \multicolumn{10}{|l|}{ Controllability } \\
\hline DM controllable by PA change & 455 & 87.0 & 139 & $84 \cdot 2$ & 316 & 88.3 & 1.41 & $0.83,2.39$ & 0.205 \\
\hline
\end{tabular}

DM, type 2 diabetes mellitus; PA, physical activity.

Data are presented as $n$ and \%.

*Six common risk factors: overweight, too little exercise, unhealthy diet, age more than 35 years, smoking and hypertension. 
Table 3 Odds ratios of association of risk perception parameters with attendance after mutual adjustment for risk perception (model 1) and corrected for potential influencing factors; Hindustani Surinamese (South Asians) aged 18-60 years participating in a diabetes lifestyle or control intervention for South Asians in The Hague, the Netherlands (DH!AAN study)

\begin{tabular}{|c|c|c|c|c|c|c|c|c|c|c|c|c|c|c|}
\hline \multirow[b]{2}{*}{ Risk perception } & \multicolumn{2}{|c|}{ Model $1^{*}$} & \multicolumn{2}{|c|}{$\begin{array}{l}\text { Model } 1+\text { Age } \\
\text { and gender }\end{array}$} & \multicolumn{2}{|c|}{$\begin{array}{l}\text { Model } 1+\text { Blood } \\
\text { test results } \\
\end{array}$} & \multicolumn{2}{|c|}{$\begin{array}{l}\text { Model } 1+ \\
\text { Attitude }\end{array}$} & \multicolumn{2}{|c|}{$\begin{array}{l}\text { Model } 1+\text { Social } \\
\text { support brown rice }\end{array}$} & \multicolumn{2}{|c|}{$\begin{array}{l}\text { Model } 1+\text { Self- } \\
\quad \text { efficacy } \ddagger\end{array}$} & \multicolumn{2}{|c|}{$\begin{array}{c}\text { Model } 1+\text { Motivated } \\
\text { to change PA }\end{array}$} \\
\hline & OR & $95 \% \mathrm{Cl}$ & OR & $95 \% \mathrm{Cl}$ & OR & $95 \% \mathrm{Cl}$ & OR & $95 \% \mathrm{Cl}$ & OR & $95 \% \mathrm{Cl}$ & OR & $95 \% \mathrm{Cl}$ & OR & $95 \% \mathrm{Cl}$ \\
\hline \multicolumn{15}{|c|}{ Causal beliefs: general lifestyle factors§ } \\
\hline $\begin{array}{l}\text { Perceived general lifestyle } \\
\text { factors as a cause } \S\end{array}$ & 1.08 & $0.70,1.67$ & 1.02 & $0.65,1.58$ & 1.09 & $0.70,1.69$ & 1.06 & $0.68,1.64$ & $1 \cdot 10$ & $0.71,1.71$ & $1 \cdot 12$ & $0.72,1.73$ & 1.09 & $0.70,1.69$ \\
\hline \multicolumn{15}{|c|}{ Causal beliefs: group-specific lifestyle factors } \\
\hline $\begin{array}{l}\text { Perceived consuming much } \\
\text { sugar as a cause }\end{array}$ & $1 \cdot 12$ & $0.73,1.72$ & $1 \cdot 20$ & $0.78,1.86$ & 1.09 & $0.71,1.69$ & $1 \cdot 11$ & $0.73,1.74$ & $1 \cdot 15$ & $0.74,1.77$ & $1 \cdot 13$ & $0.73,1.75$ & $1 \cdot 16$ & $0.75,1.79$ \\
\hline Perceived white rice as a cause & 0.89 & $0.59,1.35$ & 0.91 & $0.60,1.38$ & 0.92 & $0.60,1.40$ & 0.84 & $0.55,1.29$ & 0.88 & $0.58,1.33$ & 0.90 & $0.59,1.36$ & 0.87 & $0.58,1.32$ \\
\hline Perceived masala as a cause & 1.08 & $0.71,1.65$ & $1 \cdot 12$ & $0.73,1.71$ & 1.07 & $0.70,1.64$ & 1.08 & $0.71,1.66$ & 1.06 & $0.69,1.61$ & 1.08 & $0.71,1.66$ & 1.06 & $0.69,1.62$ \\
\hline \multicolumn{15}{|l|}{ Causal beliefs: heredity } \\
\hline $\begin{array}{l}\text { Perceived being South Asian as } \\
\text { a cause }\end{array}$ & 1.02 & $0.65,1.59$ & 1.08 & $0.69,1.70$ & 1.02 & $0.65,1.60$ & 0.98 & $0.62,1.54$ & 0.98 & $0.63,1.54$ & 1.03 & $0.65,1.61$ & 1.02 & $0.65,1.60$ \\
\hline $\begin{array}{l}\text { Perceived family history of DM } \\
\text { as a cause }\end{array}$ & 1.58 & $0.88,2.86$ & 1.60 & $0.88,2.92$ & 1.47 & $0.80,2.67$ & 1.66 & $0.91,3.03$ & 1.60 & $0.88,2.91$ & 1.61 & $0.89,2.92$ & 1.55 & $0 \cdot 86,2 \cdot 82$ \\
\hline \multicolumn{15}{|l|}{ Perceived susceptibility } \\
\hline $\begin{array}{l}\text { Perceived themselves as } \\
\text { susceptible }\end{array}$ & 1.39 & $0.94,2.05$ & 1.32 & $0.89,1.96$ & 1.33 & $0.90,1.97$ & 1.40 & $0.94,2.08$ & 1.38 & $0.93,2.04$ & $1 \cdot 31$ & $0.88,1.94$ & 1.35 & $0.91,1.99$ \\
\hline \multicolumn{15}{|l|}{ Perceived controllability } \\
\hline DM controllable by PA change & 1.34 & $0.77,2.33$ & 1.41 & $0.81,2.47$ & 1.40 & $0.80,2.44$ & NAll & NAll & $1 \cdot 30$ & $0 \cdot 75,2 \cdot 27$ & 1.41 & $0.81,2.47$ & 1.28 & $0.73,2 \cdot 24$ \\
\hline
\end{tabular}

DM, type 2 diabetes mellitus; PA, physical activity; NA, not applicable.

Data are OR and $95 \%$ Cl of the parameter of risk perception in the indicated model.

Ther the

were included in the univariate analyses; i.e. direct and indirect attitudes towards PA and the importance of replacing white rice with brown rice.

Only the parameters of perceived self-efficacy with $P<0.25$ were included in the univariate analyses; i.e. perceived self-efficacy for changing PA, adoption of a conventional healthy diet, and replacing white rice with sSix common

on risk factors: overweight, too little exercise, unhealthy diet, age more than 35 years, smoking and hypertension.

Note: perceived controllability by PA change was also one of the seven variables included in the indirect attitude towards PA and is therefore closely correlated with this variable. 
than that of our population. In spite of these educational differences, group-specific lifestyle factors were frequently perceived as causes of diabetes in both studies ${ }^{(22)}$. This indicates that cultural beliefs may influence the causal beliefs in our South Asian population.

Although we expected many participants to have a higher perceived susceptibility due to a high awareness and possible feelings of inevitability caused by the high prevalence of diabetes in their families, we found a relatively low prevalence of perceived susceptibility in our population. This low prevalence of perceived susceptibility was in line with previous findings ${ }^{(8,13,39-43)}$. As in other populations at high risk of diabetes, a plausible explanation for the low prevalence of perceived susceptibility in our South Asian population is unrealistic optimism, as in Weinstein's theory ${ }^{(13,44)}$, which states that some people believe that they are healthier than others, often because they do not correctly apply their knowledge about the main risk factors to themselves.

Despite the fact that several participants in our study reported being aware of the general lifestyle beliefs, perceiving themselves susceptible and perceiving diabetes to be controllable by PA, we did not find that these participants were more likely to attend the diabetes prevention programme than those who did not report this. In addition, we did not find that the group-specific lifestyle beliefs were associated with attendance.

We had expected that the heredity beliefs would have been associated with attendance. Nevertheless, the perceived influence of having a family history of diabetes was not significantly associated with attendance after adjustment for relevant confounders. This suggests that the heredity beliefs in our population are not strong triggers for participation.

This lack of association between the other parameters of risk perception and attendance contrasts with previous work in the general population that found an association of general lifestyle beliefs, perceived susceptibility and perceived controllability with attendance ${ }^{(7,8,45)}$. The lack of association of general lifestyle beliefs and perceived susceptibility with attendance might be related to a limitation in the data collection tools used. For instance, we only used a more general measure rather than a personalized one. A more personalized measure in which the awareness of someone's own health is taken into account might be a better predictor. For instance, Toft and colleagues measured both general perceived susceptibility and perceived susceptibility based on the participant's current behaviour (e.g. 'Do you think your dietary habits increase your risk of cardiovascular disease?') and found that only susceptibility linked to the participant's current dietary behaviour was positively associated with attendance after correction for confounding ${ }^{(8)}$. In addition, Cooper and colleagues used the Illness Perception Questionnaire and found an association between lifestyle causal beliefs and attendance. This questionnaire seemed to place more emphasis on the relationship between the participant's own behaviour and the illness state ${ }^{(45)}$.

Our population more frequently reported perceived controllability of diabetes than other populations at risk of diabetes ${ }^{(40,46,47)}$. Yet, the lack of association with controllability might be an indication that the role of perceived controllability is different among South Asians than among other populations. However, we wish to be cautious because we included only one measure of perceived controllability and it focused on PA, while our lifestyle intervention also focused on diet.

\section{Limitations}

Our study has several limitations. First, the enrolment in the intervention trial (including screening) was partly selective. The participants tended to have a higher level of education than the eligible non-participants of the intervention trial and to have a family history of diabetes. Such selective participation has also been observed in other studies $^{(48-50)}$. As these parameters have been shown to be associated with higher risk perception, our reported risk perception may have been overestimated. However, given the lack of association between the measured risk perception parameters and attendance, we do not believe that the reported association was affected.

Second, we measured risk perception after the results of the initial screening had been communicated to the participants. This personal risk communication may have positively affected the risk perception. Although this may have influenced the generalizability of our findings concerning risk perception, this limitation would not have influenced the association of risk perception and attendance itself.

Third, we did not measure the perceived consequences of diabetes, which is also a recognized aspect of risk perception. We cannot rule out the fact that this factor might have influenced attendance in our population, although previous studies do not show an association between perceived consequences of diabetes and attendance $^{(7,8)}$. Similarly, as already mentioned, we only measured the perceived controllability by PA and not by diet.

Finally, we were unable to account for family influences in our analyses although family members may increase social support, resulting in a positive influence on attendance at an intervention ${ }^{(32)}$. Moreover, we did not take into account other factors that might have contributed to attendance at the diabetes prevention programme. For instance, distance from the intervention location or depression may have influenced attendance ${ }^{(7,32)}$.

\section{Conclusions}

In summary, our study is the first to analyse the role of risk perception in attendance at a lifestyle intervention for a population at high risk of diabetes. Our 18- to 60-year-old South Asian population, identified by screening as being at 
high risk of diabetes, frequently reported lifestyle and heredity as causes of diabetes and reported a high perceived controllability of diabetes by PA. However, most did not perceive themselves as susceptible to diabetes. The risk perception parameters were not significantly associated with attendance at the lifestyle and control interventions. This suggests that strategies to increase general measures of risk perception may have little influence on this high-risk population's attendance. Although attendance might be increased by making the risk information during recruitment more personalized, our data suggest that future research should determine whether there are determinants other than risk perception (e.g. attitude or subjective norm) that might more effectively enhance this population's attendance.

\section{Acknowledgements}

Financial support: This study was funded by the Netherlands Organization for Health Research and Development (ZonMW; grant number 120620023). ZonMW had no role in the design, analysis or writing of this article. Conflicts of interest: None. Authorship: E.M.A.V. analysed the data, contributed to the interpretation and drafted the manuscript. B.J.C.M., V.N., M.N. and K.S. were consulted for the design and interpretation and reviewed the manuscript. I.G.M.v.V. contributed to the design of the study, interpretation of the data and reviewed/edited the manuscript. Ethics of human subject participation: The Medical Ethics Committee of the Academic Medical Center of Amsterdam, the Netherlands, has approved the study.

\section{Supplementary material}

To view supplementary material for this article, please visit http://dx.doi.org/10.1017/S1368980014001086

\section{References}

1. Wild S, Roglic G, Green A et al. (2004) Global prevalence of diabetes: estimates for the year 2000 and projections for 2030. Diabetes Care 27, 1047-1053.

2. Kanaya AM, Wassel CL, Mathur D et al. (2010) Prevalence and correlates of diabetes in South Asian Indians in the United States: findings from the metabolic syndrome and atherosclerosis in South Asians living in America study and the multi-ethnic study of atherosclerosis. Metab Syndr Relat Disord 8, 157-164.

3. Gholap N, Davies M, Patel K et al. (2011) Type 2 diabetes and cardiovascular disease in South Asians. Prim Care Diabetes 5, 45-56.

4. Bindraban NR, van Valkengoed IG, Mairuhu G et al. (2008) Prevalence of diabetes mellitus and the performance of a risk score among Hindustani Surinamese, African Surinamese and ethnic Dutch: a cross-sectional population-based study. BMC Public Health 8, 271.

5. Nicolaou M, Vlaar EMA, van Valkengoed IG et al. (2013) Development of a diabetes prevention program for Surinamese South Asians in the Netherlands. Health Promot Int (Epublication ahead of print version).
6. Douglas A, Bhopal RS, Bhopal R et al. (2011) Recruiting South Asians to a lifestyle intervention trial: experiences and lessons from PODOSA (Prevention of Diabetes \& Obesity in South Asians). Trials 12, 220.

7. Wyer S, Joseph S \& Earli L (2001) Predicting attendance at cardiac rehabilitation: a review and recommendations. Coronary Health Care 5, 171-177.

8. Toft UN, Kristoffersen LH, Aadahl M et al. (2007) Diet and exercise intervention in a general population-mediators of participation and adherence: the Inter99 study. Eur J Public Health 17, 455-463.

9. Cameron LD (2003) Conceptualizing and assessing risk perceptions: a self-regulatroy perspective. Presented at the Conceptualizing and Measuring Risk Perceptions Workshop, Washington, DC, 13-14 February 2003; availabe at http://cancercontrol.cancer.gov/brp/presentations/cameron. pdf

10. Drottz-Sjöberg B-M (1991) Perception of Risk. Studies of Risk Attitudes, Perceptions and Definitions. Stockholm: School of Economics, Center for Risk Research.

11. Dorman JS, Valdez R, Liu T et al. (2012) Health beliefs among individuals at increased familial risk for type 2 diabetes: implications for prevention. Diabetes Res Clin Pract 96, 156-162.

12. Hivert MF, Warner AS, Shrader P et al. (2009) Diabetes risk perception and intention to adopt healthy lifest yles among primary care patients. Diabetes Care 32, 1820-1822.

13. Claassen L, Henneman L, Nijpels G et al. (2011) Causal beliefs and perceptions of risk for diabetes and cardiovascular disease, The Netherlands, 2007. Prev Chronic Dis 8, A130.

14. Claassen L, Henneman L, van der Weijden T et al. (2012) Being at risk for cardiovascular disease: perceptions and preventive behavior in people with and without a known genetic predisposition. Psychol Health Med 17, 511-521.

15. Pijl M, Timmermans DR, Claassen L et al. (2009) Impact of communicating familial risk of diabetes on illness perceptions and self-reported behavioral outcomes: a randomized controlled trial. Diabetes Care 32, 597-599.

16. Marteau TM \& Lerman C (2001) Genetic risk and behavioural change. BMJ 322, 1056-1059.

17. Harwell TS, Dettori N, Flook BN et al. (2001) Preventing type 2 diabetes: perceptions about risk and prevention in a population-based sample of adults $\geq 45$ years of age. Diabetes Care 24, 2007-2008.

18. Pierce M, Harding D, Ridout D et al. (2001) Risk and prevention of type II diabetes: offspring's views. Br J Gen Pract 51, 194-199.

19. Marteau TM \& Weinman J (2006) Self-regulation and the behavioural response to DNA risk information: a theoretical analysis and framework for future research. Soc Sci Med $\mathbf{6 2}$, $1360-1368$.

20. Senior V, Marteau TM \& Peters TJ (1999) Will genetic testing for predisposition for disease result in fatalism? A qualitative study of parents responses to neonatal screening for familial hypercholesterolaemia. Soc Sci Med 48, 1857-1860.

21. Lawton J, Ahmad N, Hanna L et al. (2008) 'We should change ourselves, but we can't': accounts of food and eating practices amongst British Pakistanis and Indians with type 2 diabetes. Ethn Health 13, 305-319.

22. Raberg Kjollesdal MK, Hjellset VT, Bjorge B et al. (2011) Perceptions of risk factors for diabetes among NorwegianPakistani women participating in a culturally adapted intervention. Ethn Health 16, 279-297.

23. Choenni C \& Harmsen C (2007) Place of birth and ethnic composition of the Surinamese in the Netherlands in Dutch. Bevolkingtrends 1, 74-78.

24. Vlaar EM, van Valkengoed IG, Nierkens V et al. (2012) Feasibility and effectiveness of a targeted diabetes prevention program for 18 to 60-year-old South Asian 
migrants: design and methods of the DH!AAN study. BMC Public Health 12, 371.

25. Leventhal H, Kelly K \& Leventhal EA (1999) Population risk, actual risk, perceived risk, and cancer control: a discussion. J Natl Cancer Inst Monogr issue 25, 81-85.

26. Lindstrom J \& Tuomilehto J (2003) The diabetes risk score: a practical tool to predict type 2 diabetes risk. Diabetes Care 26, 725-731.

27. Kohinor MJ, Stronks K, Nicolaou M et al. (2011) Considerations affecting dietary behaviour of immigrants with type 2 diabetes: a qualitative study among Surinamese in the Netherlands. Ethn Health 16, 245-258.

28. Rosenstock IM (1982) The health belief model and nutrition education. J Can Diet Assoc 43, 184-192.

29. Ajzen I (2011) The theory of planned behaviour: reactions and reflections. Psychol Health 26, 1113-1127.

30. Claassen L (2011) The risky self. How people perceive and respond being at risk for diabetes and cardiovascular disease; the role of genetic risk information and self-concept. $\mathrm{PhD}$ Thesis, EMGO Institute of the VU Medical Center.

31. Lindstrom D, Sundberg-Petersson I, Adami J et al. (2010) Disappointment and drop-out rate after being allocated to control group in a smoking cessation trial. Contemp Clin Trials 31, 22-26.

32. Aggarwal B, Liao M, Allegrante JP et al. (2010) Low social support level is associated with non-adherence to diet at 1 year in the Family Intervention Trial for Heart Health (FIT Heart). J Nutr Educ Behav 42, 380-388.

33. Matthews DR, Hosker JP, Rudenski AS et al. (1985) Homeostasis model assessment: insulin resistance and $\beta$-cell function from fasting plasma glucose and insulin concentrations in man. Diabetologia 28, 412-419.

34. American Diabetes Association (2007) Diagnosis and classification of diabetes mellitus. Diabetes Care 30, Suppl. 1, S42-S47.

35. Dornelas EA, Stepnowski RR, Fischer EH et al. (2007) Urban ethnic minority women's attendance at health clinic vs. church based exercise programs. J Cross Cult Gerontol 22, 129-136.

36. Klassen AC, Garrett-Mayer E, Houts PS et al. (2008) The relationship of body size to participation and success in a fruits and vegetables intervention among low-income women. J Community Health 33, 78-89.

37. Hickson M, Macqueen C \& Frost G (2009) Evaluation of attendance and weight loss in an intensive weight management clinic compared to standard dietetic care. J Hum Nutr Diet 22, 72-76.
38. Gatewood JG, Litchfield RE, Ryan SJ et al. (2008) Perceived barriers to community-based health promotion program participation. Am J Health Behav 32, 260-271.

39. Whitford DL, McGee H \& O'Sullivan B (2009) Reducing health risk in family members of patients with type 2 diabetes: views of first degree relatives. BMC Public Health 9, 455 .

40. Harrison TA, Hindorff LA, Kim H et al. (2003) Family history of diabetes as a potential public health tool. Am J Prev Med 24, 152-159.

41. Adriaanse MC, Snoek FJ, Dekker JM et al. (2003) Perceived risk for Type 2 diabetes in participants in a stepwise population-screening programme. Diabet Med 20, 210-215.

42. Graham GN, Leath B, Payne K et al. (2006) Perceived versus actual risk for hypertension and diabetes in the African American community. Health Promot Pract 7, 34-46.

43. Jones EJ, Roche CC \& Appel SJ (2009) A review of the health beliefs and lifestyle behaviors of women with previous gestational diabetes. J Obstet Gynecol Neonatal Nurs 38, 516-526.

44. Weinstein ND (1987) Unrealistic optimism about susceptibility to health problems: conclusions from a communitywide sample. J Behav Med 10, 481-500.

45. Cooper A, Lloyd G, Weinman J et al. (1999) Why patients do not attend cardiac rehabilitation: role of intentions and illness beliefs. Heart 82, 234-236.

46. Pijl M, Henneman L, Claassen L et al. (2009) Family history of diabetes: exploring perceptions of people at risk in the Netherlands. Prev Chronic Dis 6, A54.

47. Petrie KJ, Weinman J, Sharpe N et al. (1996) Role of patients' view of their illness in predicting return to work and functioning after myocardial infarction: longitudinal study. BMJ 312, 1191-1194.

48. El Fakiri FF, Bruijnzeels MA, Foets MM et al. (2008) Different distribution of cardiovascular risk factors according to ethnicity: a study in a high risk population. J Immigr Minor Health 10, 559-565.

49. Greenland P, Hildreth NG \& Maiman LA (1992) Attendance patterns and characteristics of participants in public cholesterol screening. Am J Prev Med 8, 159-164.

50. Blackwell CS, Foster KA, Isom S et al. (2011) Healthy living partnerships to prevent diabetes: recruitment and baseline characteristics. Contemp Clin Trials 32, 40-49.

51. Prochaska JO \& DiClemente CC (1984) Self change processes, self efficacy and decisional balance across five stages of smoking cessation. Prog Clin Biol Res 156, $131-140$ 